Modern Physics Letters A

(C) World Scientific Publishing Company

\title{
Modifications to the Hadronic Regge Trajectories
}

\author{
Akhilesh Ranjan \\ Department of Physics, Manipal Institute of Technology, Manipal, Udupi, 576104, Karnataka, \\ India \\ akranjanji@rediffmail.com \\ Hemwati Nandan \\ Department of Physics, Gurukula Kangri Vishwavidyalaya, Haridwar, 249404, Uttarakhand, \\ India \\ hntheory@yahoo.co.in
}

Received (Day Month Year)

Revised (Day Month Year)

\begin{abstract}
The effect of quark mass on the Regge trajectory is analysed. Modifications in the equations of Regge trajectories are shown for mesonic as well as baryonic systems. For mesonic systems, the Regge trajectories get modified, but still remain linear. Contrary to the mesonic case, the Regge trajectories for baryonic systems indicate non-linearity. It is shown that in low mass and angular momentum region two hadrons with different quark compositions can have same mass and angular momentum.
\end{abstract}

Keywords: Regge trajectory; meson; baryon.

PACS Nos.: 11.55Jy, 14.20.-c, 14.40.-n.

\section{Introduction}

All the known hadrons are composed of quarks with three different colors as indicated by the deep inelastic scattering experiments 1 . The isolated colored quarks have not been observed in nature, i.e., they are confined to the interior of hadrons 112 which leads to the well-known problem of quark confinement. The issue of confinement is still a very challenging problem to solve in theoretical physics. This is mainly because of the highly non-perturbative nature of strong interactions in low energy (infra-red) region 2/3|4|5. In this context, it is worth to notice the string model of confined quarks in hadrons resulting from the dual resonance model 617 . Such strings are characterised by the Regge trajectories of hadrons (i.e., the appropriate relationship between the classical mass $M$ and spin $J$ of hadrons). Similar picture of the string models from the view point of the linearly rising Regge trajectories also emerges in dual superconductor models of QCD 8|9|10.

The original motivation behind the Regge theory 1112 was to prove the validity of the Mandelstam representation $13[14$ in non-relativistic potential scattering. The 
Regge representation was assumed to hold in relativistic region extended to the Mandelstam domain of analyticity for scattering amplitude in high energy hadronhadron scattering. The moving Regge poles were defined as 'Regge trajectory' and soon a variety of Regge trajectories were found to be nicely fitted to the observed hadrons and resonances in terms of well known Chew-Frautschi plots 15 . The quark bound states have linearly rising Regge trajectories with numerous possible angular momentum states for a given energy and offer crucial insight into the mechanism of the quark confinement $25 \mid 16$.

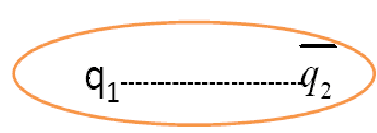

(a)

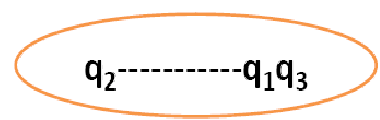

(c)

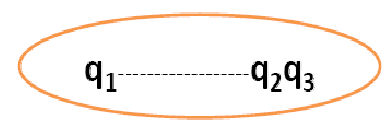

(b)

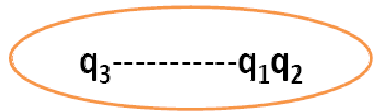

(d)

Fig. 1. (a) String model representation of meson; (b),(c) \& (d) different possible configurations of a baryon in string model.

The observed relation between the angular momentum quantum number and the energy of a particular hadronic state is given by,

$$
J=\alpha^{\prime} M^{2}+\alpha_{0}
$$

where, $\alpha_{0}$ is a constant known as the Regge intercept and $\alpha^{\prime}$ is the Regge slope parameter which is given by $\alpha^{\prime}=1 /(2 \pi K)$ here, $K$ is the linear energy density of the string. The Eq(1) holds for the case of constant energy density $K$ of the string 16 , that is, to a linear potential of the form $V(r)=K r$ where, $r$ is the interquark distance. The linearly rising Regge trajectories are, therefore, relevant to the linear form of QCD potential and again confirm the validity of the string model of hadrons. The observed value of Regge slope parameter $\left(\alpha^{\prime}=0.93 \mathrm{GeV}^{-2}\right)$ then leads $K=0.87 \mathrm{GeV} / \mathrm{fm}$ and the same result also comes from the consideration of the measured size of hadrons in electron scattering experiments 17 .

The microscopic understanding of the generic properties of the Regge trajectories for hadrons are still not completely explained. The study of the Regge trajectories 11|12|18 may, therefore, be a crucial test for the physical realisation of the string model. The total angular momentum and classical mass of the strings for mesons and baryons (or hadrons) need careful attention especially in view of the universality of the Regge slope parameter of hadronic trajectories 2. Besides the string model, there exists quark-diquark model for baryons 19|20|21/22|23 and it would also be meaningful to work out the structure of Regge trajectories in the framework of such model. In recent years, many attempts have been made to address the problem 
specially the emergence of non-linearity in the Regge trajectories with their physical realisation in different models $18|24| 25|26| 27|28| 29 \mid 30$.

In this letter, we have analysed a more realistic string model of hadrons by considering the modifications in Regge trajectories due to the finite quark mass. It is shown that the linearity of the Regge trajectories remain intact in case of mesons while the Regge trajectories for baryons deviate from linearity by deriving the expressions for the classical mass and classical angular momentum in both cases.

\section{Calculations}

In string model of hadrons, the quarks are generally assumed to be massless and spinless. In usual Regge trajectories the quark-antiquark which are connected by a string of length $l$, assumed to be rotating with the speed of light. In such case, the relation between the classical mass and classical angular momentum of a hadron is given by Eq(1).

We propose the following modifications for mesonic as well as baryonic Regge trajectories separately in view of the string model configurations presented in Fig

\subsection{Corrections for Mesonic Regge trajectories}

Let us consider, a general case where a meson is made of two different flavors of quark and antiquark with finite mass. We assume that the quark and antiquark are sitting at the opposite ends of the string. Further, we do not consider the spin of the quarks. The mesonic string is rotating about its center of mass (see Fig 1(a)). Let the quark with mass $m_{1}$ is rotating with speed $f c$ where $0<f \leq 1$ and $c$ is the speed of light in vacuum. Throughout our calculations, we have considered the natural units, i.e., $c=1$ or, $f c=f$. The mass of antiquark is $m_{2}$ and $l$ is length of the string. The modified mass of the meson $\left(M_{m o d}\right)$ is then given by

$$
M_{\text {mod }}=\frac{K m_{2} l}{f\left(m_{1}+m_{2}\right)}\left(\int_{0}^{f} \frac{d v}{\sqrt{1-v^{2}}}+\int_{0}^{\frac{m_{1}}{m_{2}} f} \frac{d v}{\sqrt{1-v^{2}}}\right)+\gamma_{1} m_{1}+\gamma_{2} m_{2}
$$

where $\gamma_{1}=\frac{1}{\sqrt{1-f^{2}}}$ and $\gamma_{2}=\frac{1}{\sqrt{1-\frac{m_{1}^{2} f^{2}}{m_{2}^{2}}}}$. After integration Eq(2), can be rewritten as

$$
M_{\text {mod }}=\frac{K m_{2} l}{f\left(m_{1}+m_{2}\right)}\left(\sin ^{-1} f+\sin ^{-1} \frac{m_{1} f}{m_{2}}\right)+\gamma_{1} m_{1}+\gamma_{2} m_{2}
$$

The modified angular momentum of meson $\left(J_{m o d}\right)$ is given by

$$
\begin{aligned}
J_{\text {mod }} & =\frac{K m_{2}^{2} l^{2}}{f^{2}\left(m_{1}+m_{2}\right)^{2}}\left(\int_{0}^{f} \frac{v^{2} d v}{\sqrt{1-v^{2}}}+\int_{0}^{\frac{m_{1}}{m_{2}} f} \frac{d v}{\sqrt{1-v^{2}}}\right) \\
& +\frac{m_{1} f l}{m_{1}+m_{2}}\left(\gamma_{1} m_{2}+\gamma_{2} m_{1}\right)
\end{aligned}
$$


and after integration which leads to the following form

$$
\begin{aligned}
J_{\text {mod }} & =\frac{K m_{2}^{2} l^{2}}{f^{2}\left(m_{1}+m_{2}\right)^{2}}\left(\frac{1}{2} \sin ^{-1} f-\frac{f}{2} \sqrt{1-f^{2}}+\frac{1}{2} \sin ^{-1} \frac{m_{1} f}{m_{2}}\right. \\
& \left.-\frac{m_{1} f}{2 m_{2}} \sqrt{1-\frac{f^{2} m_{1}^{2}}{m_{2}^{2}}}\right)+\frac{m_{1} f l}{m_{1}+m_{2}}\left(\gamma_{1} m_{2}+\gamma_{2} m_{1}\right)
\end{aligned}
$$

Now the modified expression of the inter-relationship between the classical mass and classical angular momentum can be expressed as

$$
\begin{aligned}
J_{\text {mod }} & =\frac{\pi}{\sin ^{-1} f+\sin ^{-1} \frac{m_{1} f}{m_{2}}}\left(1-\frac{\sin \left(2 \sin ^{-1} f\right)+\sin \left(2 \sin ^{-1} \frac{m_{1} f}{m_{2}}\right)}{2\left(\sin ^{-1} f+\sin ^{-1} \frac{m_{1} f}{m_{2}}\right)}\right) \\
& \times \alpha^{\prime}\left(M-\gamma_{1} m_{1}-\gamma_{2} m_{2}\right)^{2} \\
& +\frac{2 \pi\left(\frac{m_{1}}{m_{2}}\right) f^{2} \alpha^{\prime}}{\sin ^{-1} f+\sin ^{-1} \frac{m_{1} f}{m_{2}}}\left(M-\gamma_{1} m_{1}-\gamma_{2} m_{2}\right)\left(\gamma_{1} m_{2}+\gamma_{2} m_{1}\right)
\end{aligned}
$$

The expressions of $J_{m o d}$ are functions of $\sin ^{-1} \frac{m_{1} f}{m_{2}}$. Since $\sin \theta \leq 1$ so $f \leq \frac{m_{2}}{m_{1}}$. Further, according to special theory of relativity $f \leq 1$. Both of these conditions should therefore satisfy simultaneously.

\subsection{Correction for baryonic Regge trajectories}

Like a string of meson, a baryon can also be described in terms of three equally probable configurations as shown in Fig 1.(b)-(d). On following the analysis of mesons, we obtain the modified expression for classical mass of a baryon for configuration (a)

$$
M_{1}=\frac{K l\left(m_{2}+m_{3}\right)}{f\left(m_{1}+m_{2}+m_{3}\right)}\left(\sin ^{-1} f+\sin ^{-1} \frac{m_{1} f}{m_{2}+m_{3}}\right)+\gamma_{0} m_{1}+\gamma_{a}\left(m_{2}+m_{3}\right)
$$

where $m_{1}, m_{2}, m_{3}$ are masses of quarks ' 1 ', '2', and ' 3 ' respectively and $\gamma_{0}=$ $\frac{1}{\sqrt{1-f^{2}}}, \& \gamma_{a}=\frac{1}{\sqrt{1-\left(\frac{m_{1} f}{m_{2}+m_{3}}\right)^{2}}}$.

All of the above configurations for a string of baryon are equally probable. Therefore the actual modified expression for mass of a baryon should be their average.

$$
\text { or, } \begin{aligned}
M_{\text {mod }} & =\frac{2 K l}{3 f} \sin ^{-1} f+\frac{K l}{3 f\left(m_{1}+m_{2}+m_{3}\right)}\left\{\left(m_{2}+m_{3}\right) \sin ^{-1} \frac{m_{1} f}{m_{2}+m_{3}}\right. \\
& \left.+\left(m_{1}+m_{3}\right) \sin ^{-1} \frac{m_{2} f}{m_{1}+m_{3}}+\left(m_{1}+m_{2}\right) \sin ^{-1} \frac{m_{3} f}{m_{1}+m_{2}}\right\} \\
& +\gamma_{0}\left(m_{1}+m_{2}+m_{3}\right)+\gamma_{a}\left(m_{2}+m_{3}\right)+\gamma_{b}\left(m_{1}+m_{3}\right) \\
& +\gamma_{c}\left(m_{1}+m_{2}\right)
\end{aligned}
$$

where we have assumed that ' $\mathrm{f}$ ' and 'l' are same in all probable configurations. It is assumed that in configuration (a) the quark ' 1 ' rotates with speed ' $\mathrm{f}$ ', in configuration (b) the quark ' 2 ' rotates with speed ' $\mathrm{f}$ ', in configuration (c) the quark ' 3 ' 
rotates with speed ' $\mathrm{f}$ ', $\gamma_{b}=\frac{1}{\sqrt{1-\left(\frac{m_{2} f}{m_{1}+m_{3}}\right)^{2}}}$, and $\gamma_{c}=\frac{1}{\sqrt{1-\left(\frac{m_{3} f}{m_{1}+m_{2}}\right)^{2}}}$.

Similarly the modified expression of angular momentum for configuration (a) is obtained as

$$
\begin{aligned}
J_{1} & =\frac{\pi}{\sin ^{-1} f+\sin ^{-1} \frac{m_{1} f}{m_{2}+m_{3}}}\left(1-\frac{\sin \left(2 \sin ^{-1} f\right)+\sin \left(2 \sin ^{-1} \frac{m_{1} f}{m_{2}+m_{3}}\right)}{2\left(\sin ^{-1} f+\sin ^{-1} \frac{m_{1} f}{m_{2}+m_{3}}\right)}\right) \\
& \times \alpha^{\prime}\left\{M_{1}-\gamma_{0} m_{1}-\gamma_{a}\left(m_{2}+m_{3}\right)\right\}^{2} \\
& +\frac{m_{1} f l}{m_{1}+m_{2}+m_{3}}\left\{\gamma_{a} m_{1}+\gamma_{0}\left(m_{2}+m_{3}\right)\right\}
\end{aligned}
$$

Again since all the configurations are equally probable therefore the actual modified expression for angular momentum associated with the baryon should be their average. The general structure of $J_{i}$ 's (where $\mathrm{i}=1,2,3$ stands for configurations a,b, and c respectively) are given by

$$
J_{i}=C_{i}^{2}\left(M_{i}-a\right)^{2}+\gamma m_{i} f l
$$

where $C_{i}$ 's are functions of ' $f$ ', ' $l$ ', and $m_{1}, m_{2}, m_{3}$. Now it can be proved that it is not possible to write the expression for $J_{m o d}$ in the form of Eq1 for the case of baryons with finite quark masses.

In the expressions of $J_{i}$ 's, we encounter terms $\sin ^{-1} \frac{m_{i} f}{\sum_{j} m_{j}-m_{i}}$ where $\mathrm{i}, \mathrm{j}$ can take

values $1,2,3$. Since $\sin \theta \leq 1$ therefore leads to the condition $f \leq \frac{\sum_{j} m_{j}}{m_{i}}-1$ which alongwith $f \leq 1$ should satisfy simultaneously.

It is clear that if one tries to write the equation of the Regge trajectory for a mesonic system in the form of Eq1, the expressions of $\alpha^{\prime}$ and $\alpha_{O}$ will also get modified and they will become functions of quark masses as well as their rotational speed.

\section{Results and discussion}

The expressions obtained for the inter-relationship between the classical mass and angular momentum for hadrons in the last section are visually presented in the Fig2 to Fig13 with different conditions on the mass of quarks and their rotational speed. In computations, we have considered $m_{1}=1.5 \mathrm{MeV}(u$ quark $), m_{2}=$ $3.0 \mathrm{MeV}(d$ quark $), m_{3}=3.0 \mathrm{MeV}(d$ quark $), K=0.2 \mathrm{GeV}^{2}$, from Particle Data Group (PDG) 31. For computation of mass of hadrons the string length $l=1 \mathrm{fm}$ is taken. In the plots mass of hadrons and corresponding angular momenta are calculated for different values of $f$ and quark mass $m_{1}$. Then Regge trajectories are plotted.

In Fig2 and Fig 3 the dependence of meson mass and angular momentum on rotational speed of a quark are shown. Fig 4 shows the mesonic Regge trajectory. All these plots show non-linear behavior quite well. It is worth noting that in Fig4 and Fig 10, the plots are parabolic. In usual Regge trajectory with the assumption 
that the quarks are massless, the inter-relationship between $J$ and $M$ is given by equation 1 such that the plot $J$ vs $M^{2}$ is a straight line while in the present case with the consideration of massive quarks, the inter-relationship between $J$ and $M$ acquires the form $J=\alpha(M-a)^{2}+(M-b)$. The plot $J$ vs $(M-a)^{2}$ will be a straight line but since we have plotted $J$ vs $M^{2}$ therefore we obtain a parabolic trajectory. Hence in these figures, the non-linearity is not definitely an artifact of the scale used.

In Fig 5 and Fig6, the dependence of meson mass and angular momentum on quark mass are shown. In these figures, the mass and angular momentum of meson first decreases with quark mass and after acquiring a minimum value it again starts increasing. In other words, these mesons with less mass and angular momentum can be described by two different kind of constituent quark-antiquarks. This leads to a confusing behavior of the Regge trajectory of less massive mesons where one mesonic mass can have two different angular momenta. But one should note that these two angular momenta are for two different mesons having same mass as explained earlier. From the $\mathrm{Eq}([6)$, it is clear that the Regge trajectory is linear, i.e., if we plot $J$ vs $(M-a)^{2}$ as explained above while the non-linear behavior of Regge trajectories is evident Fig7 Since the linearly rising Regge trajectories are relevant to the linear form of confinement potential, it therefore remains to see the effect of the above mentioned non-linearity in Regge trajectories on the confinement potential at large distances in hadronic sense to check the validity of the string model of hadrons.

Let us compare Fig2 with Fig8 Fig 3 with Fig9 and so on. One may notice that there are similarities and it is because for meson and baryon, we have analysed the similar kind of structures (i.e., string model) as shown in Fig1, Therefore their physical interpretaion should remain same in both the cases. Hence in the Fig8 to Fig13 which describe the string model results and the Regge trajectories for baryons, the same pattern like of mesons is evident. For instance, we have also compared the nucleon resonance data $N^{*}\left(1238, \frac{3}{2}^{+}\right), N^{*}\left(1688, \frac{5}{2}^{+}\right), N^{*}\left(1920, \frac{7}{2}\right), N^{*}\left(2190, \frac{9}{2}\right)$, and $N^{*}\left(2360, \frac{11}{2}\right) 1613132$ with our calculations corresponding to baryonic Regge trajectories and found them in close agreement as shown in Fig10.

\section{Conclusions and future scope}

In this article, our primary purpose has been to see the effect of quark masses on Regge trajectories of hadrons. We have also shown, in the cases of mesons and baryons, how the Regge trajectories change with the variation of the mass of quarks as well as their rotational speed. The important conclusions drawn from this study are summarised as follows. The consideration of the massive quarks in the string model of hadrons, the expressions for the classical mass and classical angular momentum and hence the Regge trajectories are modified. For mesons, the form of the Regge trajectory (i.e., their linear behavior) remains same, but for the baryons the linearity is altered significantly. For mesonic systems, they still show linear nature but for baryonic systems they have highly non-linear behavior as presented in the 
corresponding figures. The parameters to characterise the Regge trajectory, i.e., $\alpha_{o}$ and $\alpha^{\prime}$ are also modified and become the quark mass dependent quantities. In low mass and angular momentum region two hadrons with different quark compositions can have same mass and angular momentum. The Regge trajectories for the present model are also compared with the trajectories corresponding to measured baryon masses in terms of the nucleon resonance data and are found in close agreement.

The deviation from the known experimental results may be due to exclusion of spin of quarks. Since the baryons are three body systems and the exact analysis for a three body system is an extremely complicated problem in itself. It might be possible that the non-linearity in baryonic Regge trajectories have a close connection with the mass difference between the quark and diquark masses in baryons in comparison to the linear mesonic trajectories with the equality of the quark and antiquark mass in mesons. This issue needs a careful attention. One so need to work out whether the non-linearity of Regge trajectories is dependent on the position-dependent quark masses. The analysis of modified Regge trajectories is an important task to study in greater detail especially from the view point of the data available for multitude of hadrons with the particle data group (PDG). We intend to report on such issues in our forthcoming communications.

\section{Acknowledgement}

AR is thankful to Gurukula Kangri Vishwavidyalaya Haridwar and Harish-Chandra Research Institute Allahabad for their hospitality where some part of this work was done. We are grateful to the people of India for their generous support for research. Authors are also thankful to the anonymous referees for their critical and valuable suggestions which helped in improving the presentation of the paper.

\section{References}

1. Robin Devenish and Amanda Cooper-Sarkar, Deep Inelastic Scattering, (Oxford University Press, Claredon, first edition, 2004).

2. Ta-Pei Cheng and Ling-Fong Li, Gauge theory of elementary particle physics, (Oxford University Press, Claredon, first edition, 2000).

3. M. Bander, Physics Reports 75 205(1981).

4. A. Smilga, Lectures on Quantum Chromodynamics, (World Scientific, Singapore, first edition, 2001).

5. H. Toki et. al (ed), Confinement 95. Proceedings, International RCNP Workshop on Color Confinement and Hadrons, Osaka, Japan, March 22-24, 1995, (World Scientific, Singapore, (1995).

6. G. Veneziano, Nuovo Cimento A 57 190(1968).

7. E. D. Giudice, P. D. Vecchia, and S. Fubini, Annals of Physics 70 378(1972).

8. Hemwati Nandan, H. C. Chandola, and H. Dehnen, Int.J.Theor.Phys. 44 457(2005), Erratum-ibid.47:1639-1640,2008.

9. H. Nandan, AIP Conf.Proc.939 16(2007).

10. H. Nandan, T. Anna and H.C. Chandola, Euro Phys. Letts. 67746 (2004).

11. T. Regge, Nuovo Cimento 14 951(1959). 
12. T. Regge, Nuovo Cimento 18 947(1960).

13. S. Mandelstam, Phys. Rev. 112 1344(1958).

14. S. Mandelstam, Phys. Rev. 166 1539(1968).

15. G. F. Chew and S. C. Frautschi, Phys. Rev. Lett. 8 41(1962).

16. D. H. Perkins, Introduction to High Energy Physics (Cambridge University Press, Cambridge, UK, fourth edition, 2000).

17. S. Bethke, Eur. Phys. J. C 64 689(2009).

18. A. E. Inopin, arXiv:hep-ph/0110160

19. D. B. Lichtenberg, W. Namgung,E. Predazzi and J. G. Wills, Phys. Rev. Lett. 48 1653(1982).

20. R. S. Kaushal and D. S. Kulshreshtha, Annals of Physics 108 198(1977).

21. V. Keiner, Phys. Rev.C54 3232(1996).

22. G. Eichmann et.al, AIP Conf. Proc. 1374 617(2010).

23. J. Ferretti, A. Vassallo, and E. Santopinto, Phys. Rev. C83 065204(2011).

24. Shuchi Bisht, Navjot Hothi, and Gaurav Bhakuni, EJTP 7 299(2010).

25. L. Burakovsky, arXiv:hep-ph/9904322

26. L. D. Soloviev, Unpublished work.

27. A. Inopin and G. S. Sharov, Phys. Rev. D 63 054023(2001), arXiv:hep-ph/9905499

28. G. S. Sharov, Phys. Rev. D 62 094015(2000), arXiv:hep:ph/0004003.

29. G. S. Sharov, arXiv:hep-ph/0311060

30. Jeanette Nguyen, Ph.D. thesis, Universiteit van Amsterdam, 2008.

31. W. M. Yao et al (Particle Data Group) J. Phys. G 33 1(2006).

32. K. Nakamura et al. (Particle Data Group), J. Phys. G 37 075021(2010). 


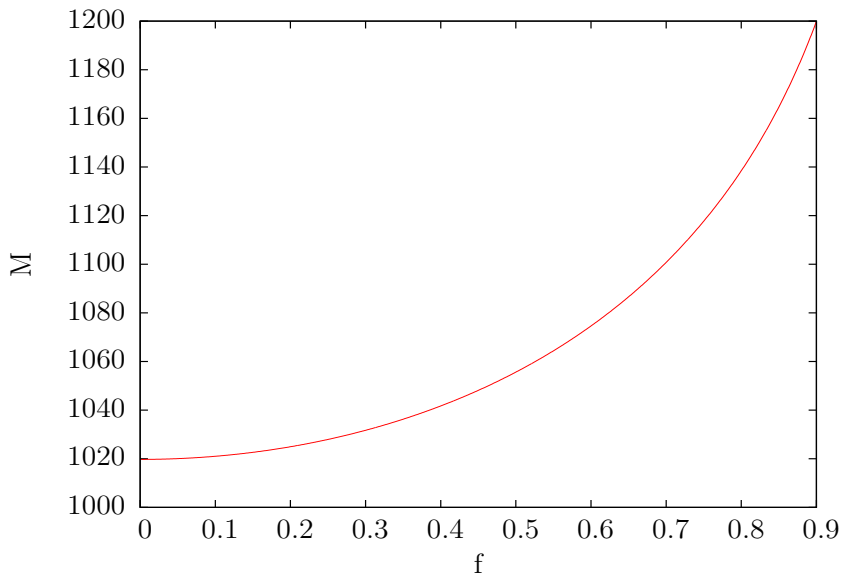

Fig. 2. Mass of meson as a function of quark 'i"s speed. Mass of quark 'i' remains fixed. 


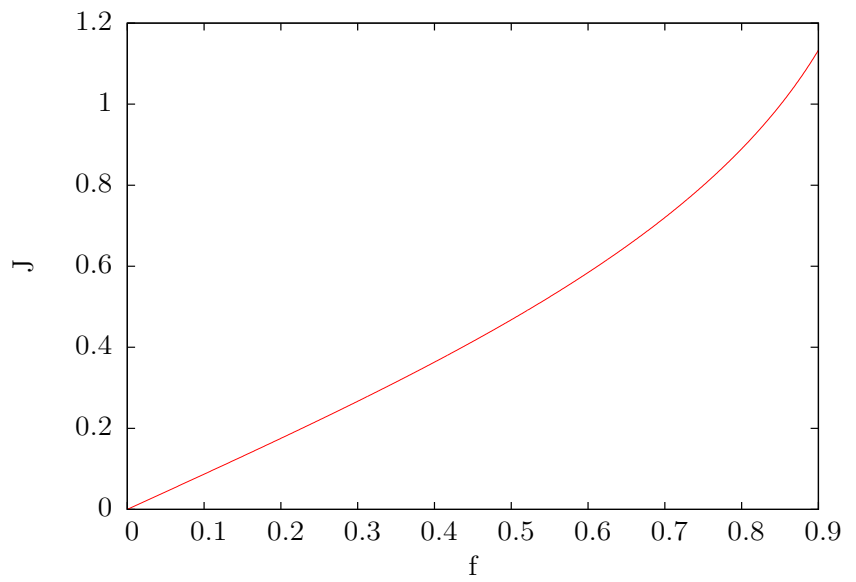

Fig. 3. Angular momentum of meson as a function of quark 'i"s speed. Mass of quark 'i' remains fixed. 


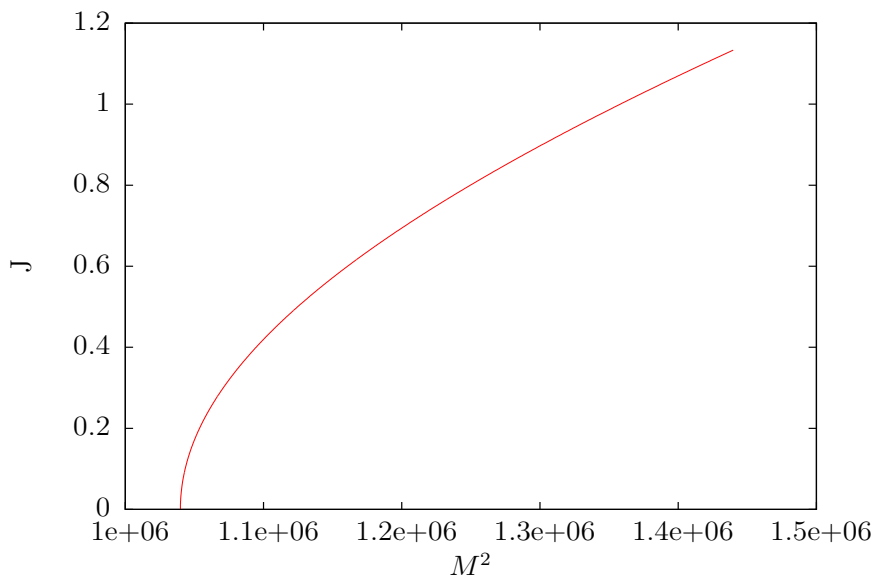

Fig. 4. The Regge trajectory for mesons when the quark 'i"s speed is varying. Mass of quark 'i' remains fixed. 


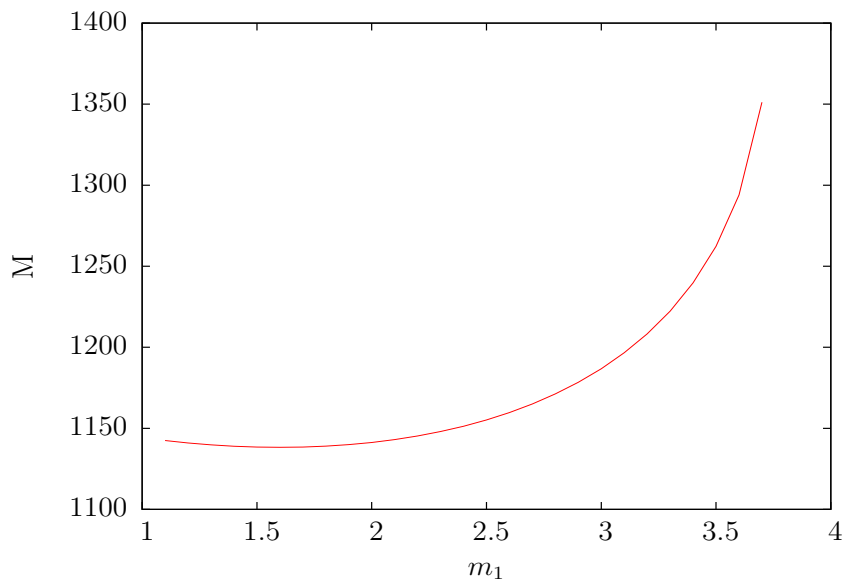

Fig. 5. Mass of meson as a function of quark 'i"s mass. Speed of quark 'i' remains fixed. 


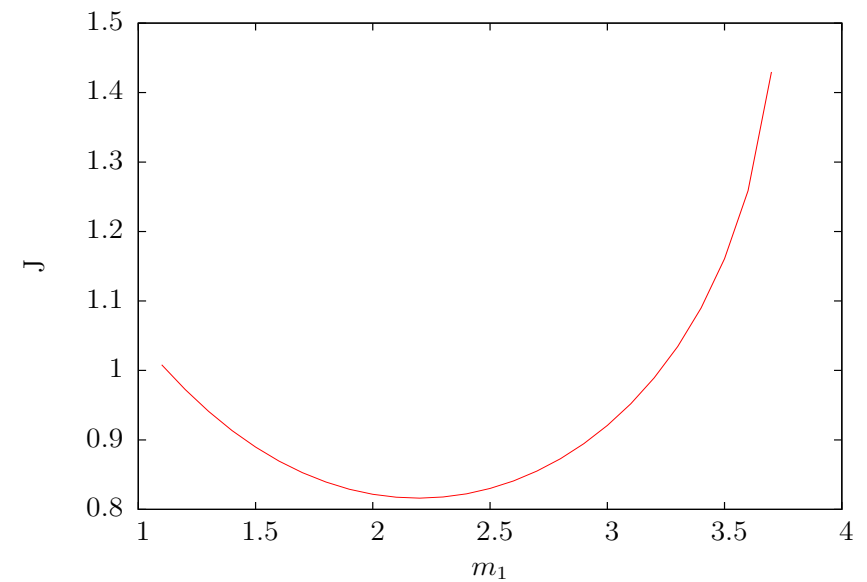

Fig. 6. Angular momentum of meson as a function of quark 'i"s mass. Speed of quark 'i' remains fixed. 


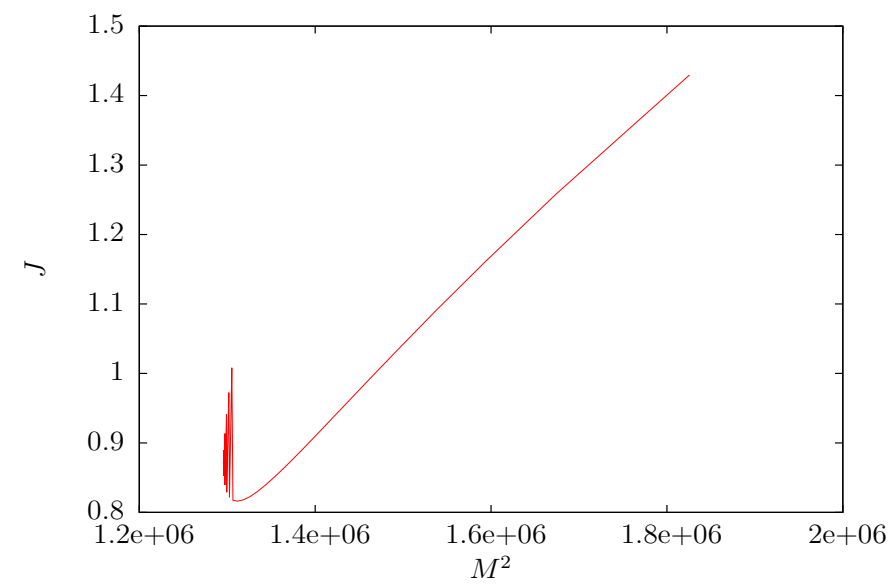

Fig. 7. The Regge trajectory for meson when the mass of quark ' $\mathrm{i}$ ' is varying. Speed of quark ' $\mathrm{i}$ remains fixed. 


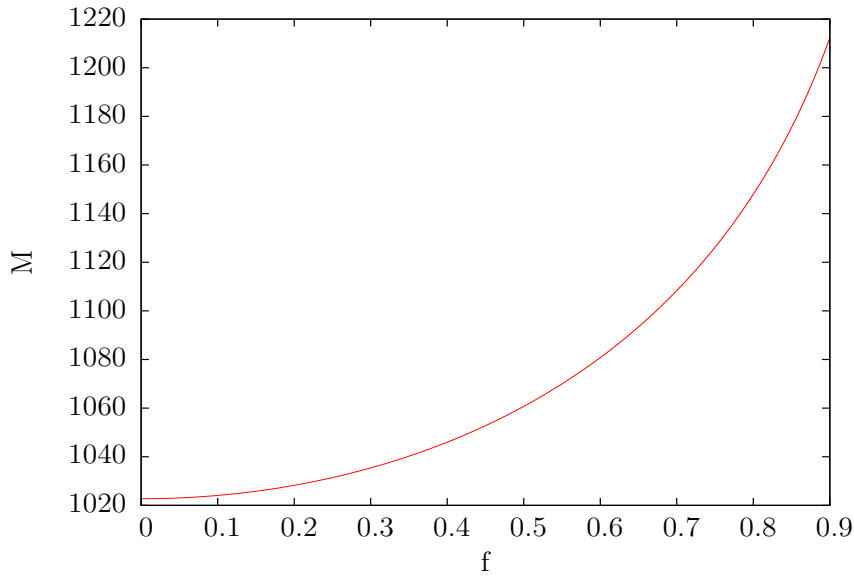

Fig. 8. Mass of baryon as a function of quark ' 1 's speed. Mass of quark ' 1 ' remains fixed. 


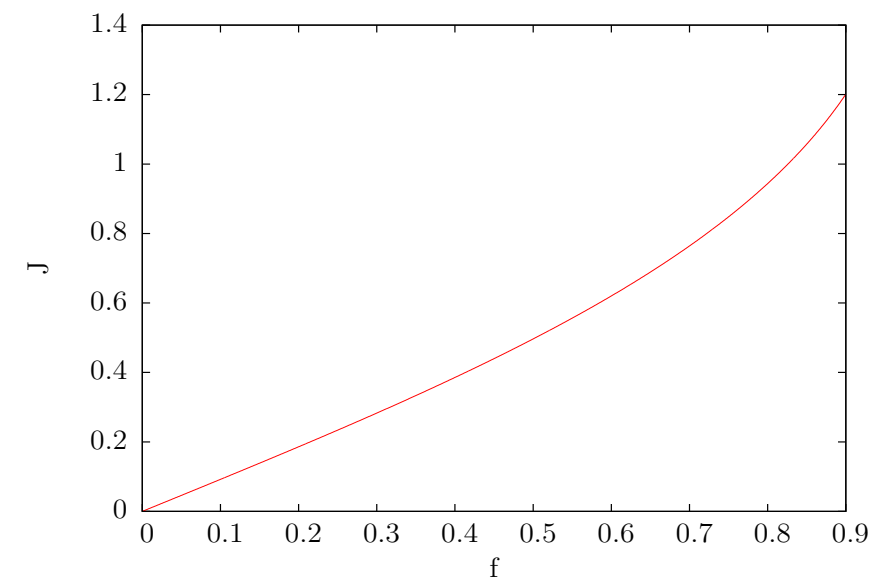

Fig. 9. Angular momentum of baryon as a function of quark ' 1 's speed. Mass of quark ' 1 ' remains fixed. 


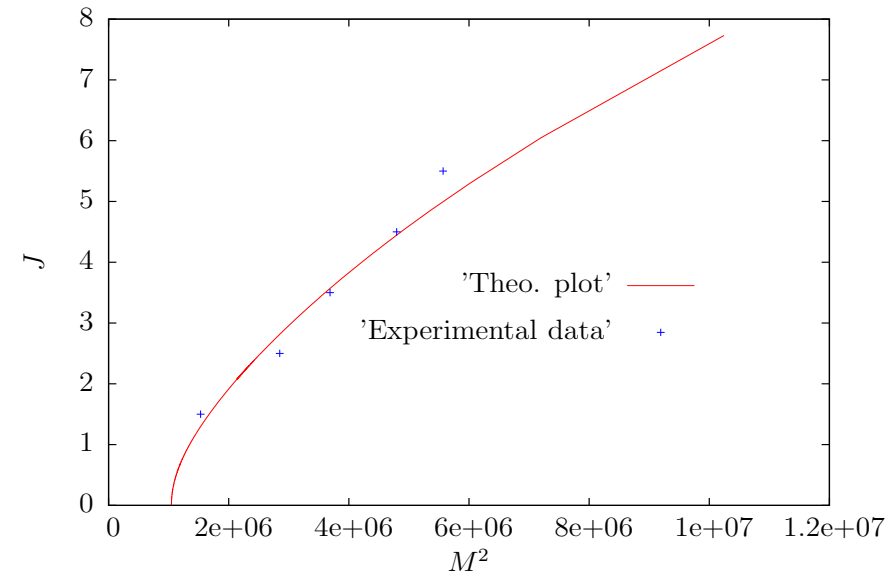

Fig. 10. The Regge trajectory for baryons when the quark '1"'s speed is varying. Mass of quark ' 1 ' remains fixed. 


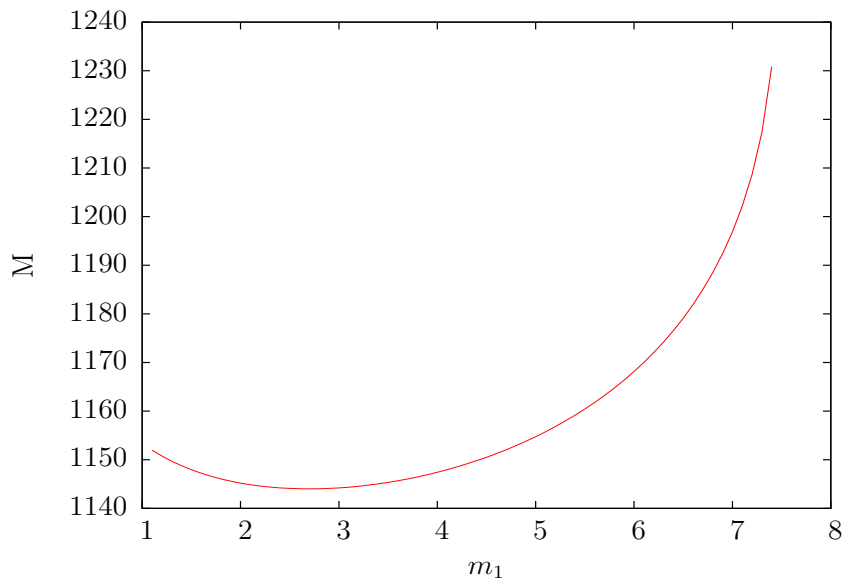

Fig. 11. Mass of baryon as a function of quark '1"'s mass. Speed of quark '1' remains fixed. 


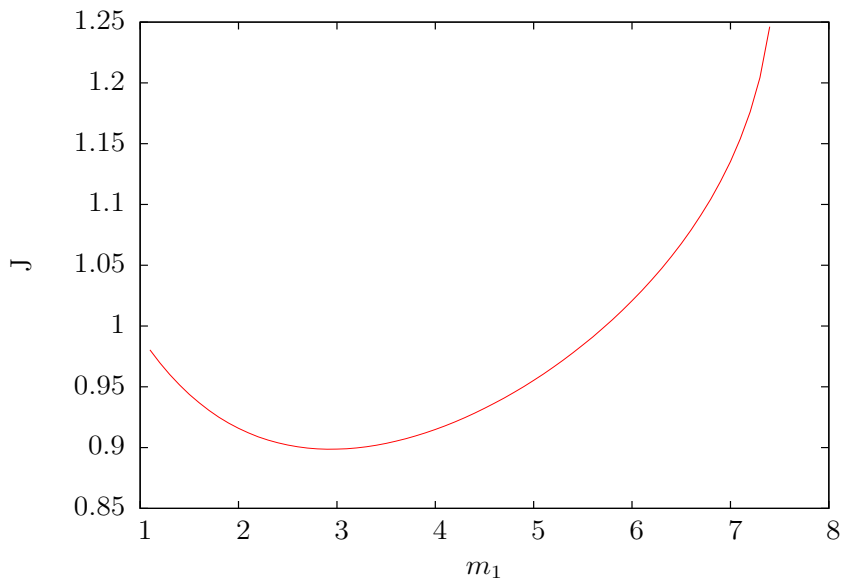

Fig. 12. Angular momentum of baryon as a function of quark '1"s mass. Speed of quark ' 1 ' remains fixed. 


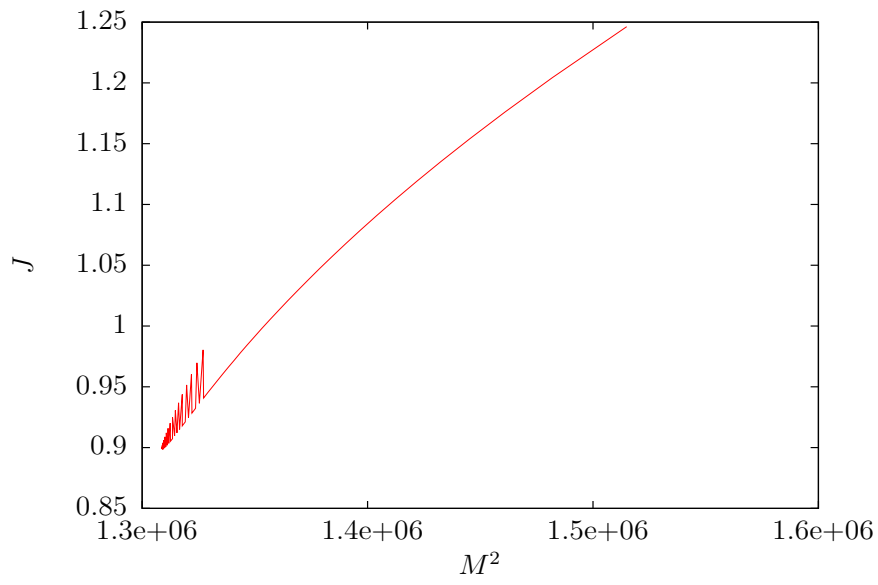

Fig. 13. The Regge trajectory for baryon when the mass of quark ' 1 ' is varying. Speed of quark ' 1 ' remains fixed. 